\title{
Modelos de Previsão de Insolvência Utilizando a Análise por Envoltória de Dados: Aplicação a Empresas Brasileiras
}

\author{
Luciana Massaro Onusic \\ Silvia Pereira de Castro Casa Nova \\ Fernando Carvalho de Almeida
}

\begin{abstract}
RESUMO
O objetivo deste artigo foi desenvolver um modelo de previsão de insolvência, utilizando uma técnica matemática originada da Pesquisa Operacional: a Análise por Envoltória de Dados (Data Envelopment Analysis - DEA). A metodologia de pesquisa foi composta pelas seguintes etapas: (1) foram selecionadas dez empresas que enfrentaram processo de falência/concordata no período de 1995 a 2003; (2) para cada empresa insolvente foram sorteadas cinco empresas entre as de melhor desempenho do setor, com porte semelhante, totalizando 50 empresas saudáveis; (3) as informações contábeis das empresas, para três anos anteriores à falência/concordata, foram obtidas no banco de dados Melhores e Maiores, de Fipecafi-Exame, e efetuadas análises estatísticas; (4) foi desenvolvido um modelo DEA e calculados os indicadores de eficiência; (5) o ponto de corte para classificação das empresas como insolventes ou solventes foi determinado; (6) as classificações obtidas pelos indicadores DEA foram confrontadas com a situação real da empresa após três anos. Os resultados preliminares mostraram que o modelo DEA desenvolvido foi capaz de discriminar, com bom grau de acerto, empresas solventes e insolventes: $90 \%$ das empresas insolventes foram corretamente classificadas.
\end{abstract}

Palavras-chave: insolvência; DEA, análise de demonstrações contábeis.

\begin{abstract}
This article aimed to develop an insolvency forecasting model through a mathematical technique that was based on Operational Research: Data Envelopment Analysis (DEA). The research methodology involved the following steps: (1) we selected ten companies that faced a bankruptcy concordat process between 1995 and 2003; (2) for each insolvent company, we chose by lot five companies among those that performed best in the sector and had a similar size, summing up a sample of 50 health companies; (3) the sample companies' accounting information for a three-year period before the bankruptcy/concordat were obtained from Fipecafi/Exame's Melhores e Maiores database, and submitted to statistical analyses; (4) we developed a DEA model and calculated efficiency indicators for the sample companies; (5) we determined the cut-off point to rank companies as insolvent or solvent; (6) the classifications obtained through the DEA indicators were confronted with the company's actual situation. Preliminary results have shown that the developed DEA model was capable of distinguishing between solvent and insolvent companies with a reasonable level of exactness: it correctly classified $90 \%$ of the insolvent companies.
\end{abstract}

Key words: insolvency; DEA; financial statements analysis. 


\section{INTRODUÇÃO}

O histórico de estudos sobre previsão de insolvência remonta ao trabalho realizado por Fitzpatrick em 1932. Utilizando métodos de observação, classificou alguns indicadores de desempenho da empresa em relação a determinado padrão, comparando-os ao longo do tempo (Fitzpatrick, 1932).

Em 1966 Beaver apresentou o primeiro estudo com a utilização de técnicas estatísticas univariadas para a previsão de falência de empresas. Altman (1968) explorou a análise discriminante multivariada, impulsionando diversos outros estudos (Elizabetsky, 1976 como citado em Silva, 1997; Kanitz, 1974; Matias, 1978; Silva, 1982 como citado em Silva, 1997). Na década de 80 foram desenvolvidos modelos de regressão logística, a começar por Ohlson (1980). Mais recentemente foram explorados os modelos baseados em redes neurais (Almeida, 1993; Bell, Ribar, \& Verchio, 1990; Tam \& Kiang, 1992).

Recentemente, alguns estudos propuseram a conjugação entre Análise por Envoltória de Dados e análise de balanços (Ceretta \& Niederauer, 2001; Fernandez-Castro \& Smith, 1994; Santos \& Casa Nova, 2005; Smith, 1990; Zhu, 2000). Outros estudos relacionaram especificamente DEA e previsão de insolvência (Almeida \& Milioni, 2000; Fernandez-Castro \& Smith, 1994; Onusic, 2004; Paradi, Asmild, \& Simak, 2004). O presente estudo explora o uso desta técnica matemática para prever a falência de empresas, desenvolvendo e testando um modelo DEA para uma amostra de empresas brasileiras.

\section{AnÁlise por Envoltória de Dados}

O histórico do método de Análise Envoltória de Dados inicia com a tese de doutoramento de Edwardo Rhodes, apresentada à Carnegie Mellow University, em 1978, sob orientação de W. W. Cooper. Com o objetivo de analisar os resultados de experimento educacional de larga escala em escolas públicas americanas (Program Follow-Through), foi desenvolvido um modelo matemático que relacionava resultados (produtos), como, por exemplo, aumento da auto-estima (medido por testes psicológicos) com insumos, como tempo gasto pelos pais em exercícios de leitura com os filhos. A tentativa de estimação da eficiência técnica de escolas com múltiplos insumos e produtos resultou na formulação do modelo CCR (abreviatura dos sobrenomes dos autores Charnes, Cooper e Rhodes) e 
com a publicação do primeiro artigo no European Journal of Operations Research, no mesmo ano (Charnes, Cooper, Lewin, \& Seiford, 1997).

Para compreensão da DEA é preciso inicialmente apresentar a nomenclatura usualmente utilizada.

- Decision Making Units (unidades decisoras). São as unidades sob avaliação. No estudo original eram escolas, mas há estudos sobre bancos, agências bancárias, programas de pós-graduação, lojas, universidades, cursos MBA, pesquisadores em Engenharia de Produção no CNPq. É apenas necessário que as unidades sejam homogêneas, isto é, obtenham os mesmos resultados (produtos), utilizando os mesmo recursos (insumos), com variação apenas de quantidade ou intensidade.

. Outputs (Produtos). Também chamados de Saídas, representam os resultados obtidos pelas DMUs. Para universidades podem ser, por exemplo, número de graduados ou número de cursos. São valores observados. Para empresas, o lucro. Para pesquisadores, número de artigos publicados.

. Inputs (Insumos). São os recursos consumidos pelas DMUs para obter os resultados (produtos) desejados. São também chamadas de Entradas. No exemplo das universidades podem ser número de professores e número de funcionários. Para empresas o Patrimônio Líquido, que representa os recursos investidos pelos sócios para obter o resultado (lucro). Para pesquisadores, número de anos de estudo.

Plano de produção. São as quantidades observadas de inputs consumidos e outputs obtidos para cada DMU.

- Indicador de eficiência. É o escore de eficiência calculado para cada DMU, considerando seu plano de produção, através de um programa de programação matemática linear (PPL). O indicador varia de 0 (máxima ineficiência) a 1 (máxima eficiência). As DMUs com indicador igual a 1 formam a fronteira de eficiência e servem de benchmark para as demais, ineficientes.

A medida de eficiência calculada pela DEA é então uma generalização de medidas usuais de produtividade que relacionem insumos consumidos e resultados obtidos, ou seja, para os exemplos citados:

Número de alunos graduados

Número de professores 
$\mathrm{Ou}$

Lucro

Patrimônio Líquido

A vantagem da DEA é a possibilidade de relacionar múltiplos produtos e múltiplos insumos em uma medida singular de eficiência, com uma compreensão intuitiva.

A formulação matemática do modelo CCR original pode ser apresentada pelas equações de 1 a 4 .

\section{Sejam:}

. $h_{k} \mathrm{o}$ indicador de eficiência da empresa k;

- $\mathrm{y}_{\mathrm{rk}}$ as quantidades observadas para os $\mathrm{r}$ tipos de produtos da empresa $\mathrm{k}$;

. $\mathrm{X}_{\mathrm{ik}}$ as quantidades observadas para os $\mathrm{i}$ tipos de insumos da empresa $\mathrm{k}$;

. $\mathrm{u}_{\mathrm{r}}$ os pesos calculados para os $\mathrm{r}$ tipos de produtos, $\operatorname{com} \mathrm{r}=1 \mathrm{a} \mathrm{m}$;

- $\mathrm{v}_{\mathrm{i}}$ os pesos calculados para os i tipos de insumos, com $\mathrm{i}=1$ a $\mathrm{n}$;

- $\mathrm{y}_{\mathrm{rj}}$ as quantidades observadas para os $\mathrm{r}$ tipos de produtos das empresas, com $\mathrm{j}=1 \mathrm{a} \mathrm{N}$;

- $\mathrm{x}_{\mathrm{ij}}$ as quantidades observadas para os $\mathrm{i}$ tipos de insumos das empresas $\mathrm{j}$, com $\mathrm{j}=1$ a N.

Maximizar $h_{k}=\sum_{r=1}^{s} u_{r} y_{r k}$

sujeito a

$\sum_{r=1}^{m} u_{r} y_{r j}-\sum_{i=1}^{n} v_{i} x_{i j} \leq 0$

$\sum_{i=1}^{n} v_{i} x_{i k}=1$

$u_{r}, v_{i} \geq 0$

O objetivo do PPL é encontrar o conjunto de pesos $u$, para os outputs, e de pesos $v$, para os inputs, que resultarão na máxima eficiência possível para a DMU sob avaliação. Dessa forma, os pesos são atribuídos a cada variável, de 
forma a colocar a unidade 'sob a melhor luz'. Como os pesos são calculados pelo PPL, a análise de eficiência é possível mesmo em situações onde não haja preços para os inputs e outputs.

O modelo será implementado para cada uma das $\mathrm{N}$ empresas, com alteração da função objetivo e calculados os indicadores de eficiência por meio da atribuição de pesos aos seus valores de produtos e insumos.

Outro modelo de DEA referenciado na literatura é chamado de BCC (Banker, Charnes, \& Cooper, 1984). O modelo BCC permite que as unidades avaliadas apresentem retornos variáveis de escala. Ou seja, que o acréscimo em uma unidade de insumo possa gerar um acréscimo não proporcional no volume de produtos. Segundo Belloni (2000) "ao possibilitar que a tecnologia exiba propriedades de retornos a escalas diferentes ao longo de sua fronteira, esse modelo admite que a produtividade máxima varie em função da escala de produção". As equações de 5 a 8 representam o modelo BCC.

\section{Sejam:}

. $\mathrm{h}_{\mathrm{k}} \mathrm{o}$ indicador de eficiência da empresa $k$;

- $\mathrm{y}_{\mathrm{rk}}$ as quantidades observadas para os $\mathrm{r}$ tipos de produtos da empresa $k$;

- $\mathrm{x}_{\mathrm{ik}}$ as quantidades observadas para os $i$ tipos de insumos da empresa $k$;

- $\mathrm{u}_{\mathrm{r}}$ os pesos calculados para os $r$ tipos de produtos, com $\mathrm{r}=1 \mathrm{a} \mathrm{m}$;

- $\mathrm{v}_{\mathrm{i}}$ os pesos calculados para os $i$ tipos de insumos, com $\mathrm{i}=1 \mathrm{an}$;

- $\mathrm{y}_{\mathrm{rj}}$ as quantidades observadas para os $r$ tipos de produtos das empresas, com $\mathrm{j}=1 \mathrm{a} \mathrm{N}$;

- $\mathrm{x}_{\mathrm{ij}}$ as quantidades observadas para os $i$ tipos de insumos das empresas $\mathrm{j}$, com $\mathrm{j}=1 \mathrm{a} \mathrm{N}$;

$\mathrm{u}_{\mathrm{k}}$ variável irrestrita, representando retornos decrescentes ou crescentes, para a empresa $\mathrm{k}$.

$$
\text { Maximizar } h_{k}=\sum_{r=1}^{m} u_{r} y_{r k}-u_{k}
$$

sujeito a

$\sum_{i=1}^{n} v_{i} x_{i k}=1$ 
$\sum_{r=1}^{m} u_{r} y_{r j}-\sum_{i=1}^{n} v_{i} x_{i j}-u_{k} \leq 0$

$u_{r}, v_{i} \geq 0$

Os modelos incorporam ainda orientação ao produto ou ao insumo. No modelo com orientação ao produto, as quantidades de produtos são maximizadas mantendose constante a quantidade consumida de insumos. No modelo com orientação ao insumo, as quantidades consumidas de insumos são minimizadas, enquanto as quantidades produzidas de recursos são mantidas constantes.

A Figura 1 representa, graficamente, os resultados obtidos em uma análise DEA hipotética. Os pontos no gráfico são as empresas sob avaliação. A curva é definida pelas empresas que obtiveram a alocação ótima entre a soma ponderada de produtos e a soma ponderada de insumos $\left(\mathrm{h}_{\mathrm{k}}\right)$, ou seja, indicador de eficiência igual a 1 .

Essas empresas são identificadas pela técnica DEA como unidades eficientes e posicionadas na curva de máxima eficiência relativa (fronteira eficiente). As demais unidades, não eficientes, estão posicionadas abaixo da curva, 'envolvidas ‘ pelo desempenho das unidades eficientes.

Figura 1: Comparação entre DEA e Análise de Regressão

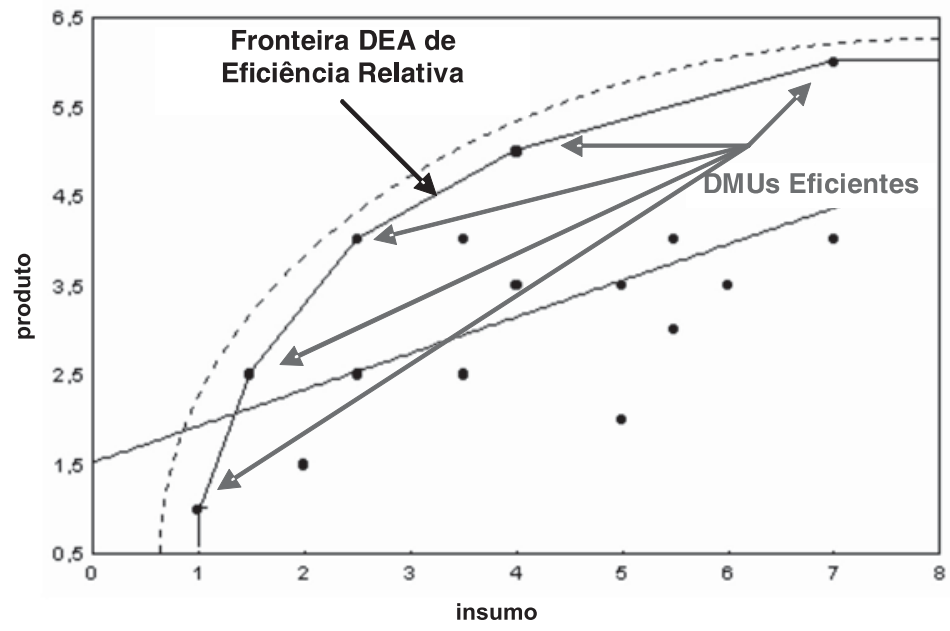

Fonte: Niederauer, Carlos Alberto Pittaluga (1998) 
Aos modelos iniciais foram acrescentados outros com possibilidades de aplicação distintas, formando uma família de modelos DEA. Os mais largamente utilizados são os modelos CCR e BCC.

\section{DEA e Análise de Balanço}

Segundo Zhu (2003), desde que a DEA foi introduzida em 1978, mais de 2000 artigos relacionados à DEA foram publicados. Os pesquisadores em diversos campos rapidamente reconheceram que a DEA é uma excelente metodologia para processos de modelagem operacional.

O primeiro trabalho relacionando DEA e análise de balanços foi apresentado por Smith (1990), que propôs, então, examinar em que extensão as limitações da análise tradicional poderiam ser superadas com a utilização da Análise por Envoltória de Dados. Posteriormente, Fernandez-Castro e Smith (1994) desenvolveram um modelo que obteve sucesso moderado em isolar empresas insolventes e propuseram sua utilização conjunta com outros instrumentos. Simak (1997) analisou a possibilidade de utilizar DEA como ferramenta para prever a insolvência corporativa futura e comparou os resultados do modelo desenvolvido com o modelo Z Score de Altman, Haldeman e Narayanan (1977). Zhu (2000) desenvolveu um modelo DEA de desempenho de empresas, utilizando o ranking da Fortune 500, aplicando-o para obter um escore de desempenho geral de empresas, com base em 8 fatores financeiros obtidos na Fortune 500. Simak (2000) apresentou metodologia que utiliza DEA para fornecer uma medida exata de avaliação de risco de crédito das empresas. Emel, Oral, Reisman e Yolalan (2003) aplicaram DEA na análise de crédito de indústrias que compunham a carteira de crédito de um dos maiores bancos comerciais da Turquia, obtendo um escore de eficiência financeira batizado de escore de credibilidade. Os resultados obtidos foram validados através de análise de regressão, análise discriminante e comparação com a opinião de analistas de crédito. Finalmente, Paradi et al. (2004) apresentaram o conceito de fronteira DEA de piores práticas, que objetivava identificar as empresas eficientes em serem ruins e posicioná-las na fronteira.

No Brasil, o estudo pioneiro relacionando DEA e Análise de Balanços foi apresentado por Ceretta em 1999. O estudo aplicou DEA em empresas do setor de alimentos brasileiro com base em dados da revista Exame - Melhores e Maiores. Mais recentemente, Almeida e Milioni (2000) propuseram um modelo de credit scoring baseado em DEA. Onusic (2004) propôs a utilização conjunta de DEA e regressão logística, testando-a em uma base de dados de empresas obtida na SERASA no período de 1995 a 2001. Finalmente, Santos e Casa Nova 
(2005) apresentaram um modelo DEA para análise de balanços e compararam seus resultados com indicador de excelência empresarial de Melhores e Maiores da revista Exame.

\section{Metodologia}

\section{Amostra e Coleta de Dados}

As empresas selecionadas para estudo compunham a base de dados da publicação 'Melhores e Maiores' da revista Exame, elaborada pela Fipecafi, Fundação Instituto de Pesquisas Contábeis, Atuariais e Financeiras, de São Paulo, Brasil. Inicialmente, foram localizadas empresas que deixaram de publicar suas demonstrações financeiras por estarem em processo de concordata ou falência. A situação de insolvência dessas empresas foi confirmada por pesquisas realizadas em jornal de grande circulação e junto ao Fórum de Falência e Concordata da Cidade de São Paulo. Foram identificados dez casos de empresas em processo de concordata ou falência para o período de 1995 a 2003. Este é, portanto, o universo de empresas localizadas nesta base de dados em situação de falência/ concordata para o período em estudo.

Para cada empresa insolvente, foram sorteadas cinco empresas-espelho (solventes), entre as melhores empresas do setor em 2003, segundo os critérios de classificação da revista, com o mesmo porte. O critério de selecionar entre as melhores empresas do setor em 2003 buscou assegurar que essas empresas tivessem boa saúde financeira nos últimos anos. Esse procedimento já havia sido adotado anteriormente no estudo de André Laredo (1997).

Assim, foi obtida uma amostra de 60 empresas, sendo dez empresas insolventes e 50 empresas-espelho (solventes), para o período em análise.

As informações contábeis das empresas foram obtidas na base de dados 'Melhores e Maiores', de Fipecafi-Exame, para três anos antes da falência/ concordata. As empresas não foram identificadas por estarem incluídas empresas de capital fechado e limitadas, que não publicam demonstrações contábeis. A Tabela 1 resume as informações das empresas selecionadas para estudo. 
Tabela 1: Resumo das Características das Empresas da Amostra

\begin{tabular}{|c|c|c|c|}
\hline Setor & $\begin{array}{l}\text { Ano da } \\
\text { concordata/ } \\
\text { falência }\end{array}$ & $\begin{array}{l}\text { Dificuldades Financeiras } \\
\text { (Ano utilizado no modelo) }\end{array}$ & $\begin{array}{l}\text { Empresas-espelho } \\
\text { (Solventes) }\end{array}$ \\
\hline Alimentos & $\begin{array}{l}2000 \\
2002\end{array}$ & $\begin{array}{l}1 \text { empresa em } 1997 \\
1 \text { empresa em } 1999\end{array}$ & $\begin{array}{l}5 \text { empresas } 1997 \\
5 \text { empresas } 1999\end{array}$ \\
\hline Química e Petroquímica & 2000 & 1 empresa em 1997 & 5 empresas 1997 \\
\hline Siderurgia e Metalurgia & 1999 & 1 empresa em 1996 & 5 empresas 1996 \\
\hline $\begin{array}{l}\text { Eletro } \\
\text { Eletrônico }\end{array}$ & 2000 & 1 empresa em 1997 & 5 empresas 1997 \\
\hline Construção & 1998 & 1 empresa em 1995 & 5 empresas 1995 \\
\hline Comércio Varejista & 2002 & 1 empresa em 1999 & 5 empresas 1999 \\
\hline Confecções e Têxtil & 2000 & 1 empresa em 1997 & 5 empresas 1997 \\
\hline Automotivo & 1998 & 1empresa em 1995 & 5 empresas 1995 \\
\hline Atacado e Comércio Exterior & 1998 & 1 empresa em 1995 & 5 empresas 1995 \\
\hline
\end{tabular}

A amostra foi considerada suficiente, tendo em vista os critérios definidos para tamanho de amostra em outros estudos sobre DEA. J. A. Fitzsimmons e M. J. Fitzsimmons (2000, p. 402) citam:

Uma questão freqüentemente argüida a respeito do tamanho da amostra diz respeito ao número de unidades de serviços que são necessárias em comparação com número de variáveis de entrada e saída selecionadas na análise. O seguinte relacionamento, associando o número de unidades de serviços $K$ utilizadas na análise e o número de tipos de entradas $N$ e saídas $M$ que estão sendo consideradas, está baseado em achados empíricos e na experiência de usuários de DEA:

$$
K \geq 2(N+M)
$$

Ou seja, para o número de variáveis indicadas nesse estudo, e explicadas no item a seguir, aplicando-se a regra, seria necessária uma amostra de, no mínimo, doze empresas. Conclui-se que a amostra, composta por 60 empresas, tem tamanho adequado para a utilização da técnica DEA.

\section{Tratamento e Seleção de Variáveis}

A dificuldade de obtenção dos dados foi um dos fatores limitadores na seleção dos indicadores para o estudo. Assim, o critério para definição dos indicadores utilizados no estudo foi sua disponibilidade para as empresas da amostra na base de dados considerada.

Os indicadores usados para desenvolvimento do modelo DEA explicitam-se em seguida. 


\section{Variáveis de Insumo}

. Endividamento Geral (Endiv. Geral): é a soma do Passivo Circulante, incluindose as duplicatas descontadas, com o Passivo Exigível a Longo Prazo dividido pelo Ativo Total Ajustado.

Endividamento de Longo Prazo (Endiv. LP): é um indicador derivado, obtido da multiplicação do Ativo Total Ajustado pelo índice de endividamento de longo prazo, sendo o resultado dividido por 100.

Composição do Endividamento (Comp. Endiv.): é o endividamento de longo prazo menos 100. É a quantidade de dívida que está no curto prazo.

\section{Variáveis de Resultado}

- Crescimento de Vendas (Cresc. Vendas): é o crescimento da receita bruta de vendas e serviços em reais, descontada a inflação média do exercício social da empresa, medida pela variação do IGP-M.

- Retorno sobre o Ativo (ROA): é composto do Lucro Líquido dividido pelo Ativo Total Ajustado.

. Giro do Ativo (GIRO): total da Receita Bruta dividido pelo Ativo Total Ajustado.

Sendo o Ativo Total Ajustado (Exame, 2003):

o total dos recursos que estão a disposição da empresa. As duplicatas descontadas não são deduzidas do Ativo Circulante. São reclassificadas no Passivo Circulante. (...) O valor é ajustado para reconhecer os efeitos inflacionários que as empresas deixaram, por imposição legal, de considerar nas demonstrações contábeis.

Foi aplicada uma transformação de escala para as variáveis que assumiam valores negativos (Crescimento de Vendas e Retorno sobre Ativo). A transformação é necessária, pois os modelos DEA não aceitam valores negativos. A literatura sugere que alguns modelos DEA possuem a propriedade de translation invariance (Ali \& Seiford, 1990; Pastor, 1996), ou seja, não têm seus resultados alterados por uma transformação de escala das variáveis. As variáveis foram transformadas pela adição de uma constante aos indicadores que assumiram valores negativos para todas as empresas em análise. 


\section{Análise de Resultados}

O modelo DEA foi aplicado aos dados para cálculo dos indicadores de eficiência das empresas da amostra. Foi escolhido o modelo DEA com minimização de inputs e retornos variáveis de escala (BCC-Insumo). Os escores de eficiência foram calculados para todas as empresas da amostra.

Para determinação do ponto de corte foi considerado o escore de eficiência que resultava na melhor discriminação entre as empresas com boa saúde e as empresas com dificuldades financeiras. Ou seja, o ponto de corte foi escolhido de maneira a minimizar os erros de classificação, conforme proposto no estudo de Simak (1997). As empresas foram primeiramente classificadas por ordem decrescente de escore de eficiência. Depois, foi verificado qual o escore que melhor segregava as empresas insolventes das empresas com boa saúde financeira. Os resultados podem ser verificados na Tabela 2.

\section{Tabela 2: Escores de Eficiência versus Situação Financeira (Em Ordem Decrescente de Escore de Eficiência)}

\begin{tabular}{|c|c|c|c|c|c|}
\hline Empresa & Escore & Grupo & Empresa & Escore & Grupo \\
\hline 23 & $100,0 \%$ & Solvente & 28 & $73,1 \%$ & Solvente \\
\hline 22 & $100,0 \%$ & Solvente & 39 & $70,0 \%$ & Solvente \\
\hline 44 & $100,0 \%$ & Solvente & 48 & $69,3 \%$ & Solvente \\
\hline 50 & $100,0 \%$ & Solvente & 24 & $69,3 \%$ & Solvente \\
\hline 56 & $100,0 \%$ & Solvente & 15 & $69,1 \%$ & Solvente \\
\hline 58 & $100,0 \%$ & Solvente & 12 & $68,8 \%$ & Solvente \\
\hline 18 & $100,0 \%$ & Solvente & 13 & $66,5 \%$ & Solvente \\
\hline 45 & $100,0 \%$ & Solvente & 57 & $64,2 \%$ & Solvente \\
\hline 26 & $100,0 \%$ & Solvente & 4 & $62,7 \%$ & Insolvente \\
\hline 11 & $100,0 \%$ & Solvente & 19 & $61,2 \%$ & Solvente \\
\hline 59 & $100,0 \%$ & Solvente & 3 & $59,1 \%$ & Insolvente \\
\hline 41 & $100,0 \%$ & Solvente & 8 & $58,2 \%$ & Insolvente \\
\hline 36 & $99,6 \%$ & Solvente & 33 & $58,0 \%$ & Solvente \\
\hline 21 & $99,5 \%$ & Solvente & 14 & $55,1 \%$ & Solvente \\
\hline 51 & $99,2 \%$ & Solvente & 53 & $52,8 \%$ & Solvente \\
\hline 40 & $98,0 \%$ & Solvente & 60 & $52,5 \%$ & Solvente \\
\hline 20 & $88,7 \%$ & Solvente & 27 & $50,3 \%$ & Solvente \\
\hline 38 & $86,3 \%$ & Solvente & 9 & $49,5 \%$ & Insolvente \\
\hline 29 & $86,3 \%$ & Solvente & 32 & $45,7 \%$ & Solvente \\
\hline
\end{tabular}


(conclusão)

Tabela 2: Escores de Eficiência versus Situação Financeira (Em Ordem Decrescente de Escore de Eficiência)

\begin{tabular}{|r|r|l|r|r|l|}
\hline Empresa & Escore & Grupo & Empresa & Escore & Grupo \\
\hline 42 & $86,0 \%$ & Solvente & 25 & $45,3 \%$ & Solvente \\
\hline 52 & $83,7 \%$ & Solvente & 2 & $44,5 \%$ & Insolvente \\
\hline 31 & $83,5 \%$ & Solvente & 1 & $44,2 \%$ & Insolvente \\
\hline 35 & $82,4 \%$ & Solvente & 49 & $43,3 \%$ & Solvente \\
\hline 54 & $82,3 \%$ & Solvente & 5 & $42,9 \%$ & Insolvente \\
\hline 43 & $80,8 \%$ & Solvente & 55 & $40,4 \%$ & Solvente \\
\hline 46 & $79,5 \%$ & Solvente & 17 & $40,2 \%$ & Solvente \\
\hline 16 & $77,9 \%$ & Solvente & 34 & $35,1 \%$ & Solvente \\
\hline 47 & $77,2 \%$ & Solvente & 10 & $24,3 \%$ & Insolvente \\
\hline 7 & $76,9 \%$ & Insolvente & 30 & $22,2 \%$ & Solvente \\
\hline 37 & $76,6 \%$ & Solvente & 6 & $16,5 \%$ & Insolvente \\
\hline
\end{tabular}

A análise dos dados revelou que entre as empresas com indicador de eficiência abaixo de 64,2\% encontravam-se 9 entre as 10 empresas insolventes da amostra: estava excluída apenas a empresa 7, com 76,9\% de indicador de eficiência. Assim, o ponto de corte definido por aquele escore determinava um percentual de acerto de $90 \%$ para as empresas concordatárias/falidas. Entre as empresas solventes, o ponto de corte determinou um erro de classificação de 26\%: 13 das 50 empresas com boa saúde financeira têm nível de eficiência menor que esse ponto. A Tabela 3 resume os resultados obtidos.

\section{Tabela 3: Resultados Obtidos}

\begin{tabular}{|l|r|}
\hline Índices de Eficiência & $\begin{array}{l}\text { Número de } \\
\text { empresas }\end{array}$ \\
\hline Eficientes $100 \%$ & 12 \\
\hline Acima de $64,2 \%$ e menores que $100 \%$ & 26 \\
\hline Abaixo e igual a $64,2 \%$ & 22 \\
\hline
\end{tabular}

Ressalte-se também que nenhuma das empresas com escore igual a $100 \%$, portanto consideradas como relativamente eficientes pelo modelo DEA, se tornaram insolventes após três anos.

A Tabela 4 mostra o percentual de acerto e erro na classificação das empresas, utilizando esse ponto de corte. 
Tabela 4: Classificação das Empresas

\begin{tabular}{|l|c|c|c|}
\hline \multirow{2}{*}{ Grupo de origem } & \multicolumn{2}{|c|}{ Classificadas como } & \multirow{2}{*}{ Total } \\
\cline { 2 - 3 } & Insolventes & Solventes & \\
\hline Insolventes & $\mathbf{9}$ & 1 & 10 \\
\hline Insolventes (\%) & $\mathbf{9 0 \%}$ & $10 \%$ & $100 \%$ \\
\hline Solventes & 13 & $\mathbf{3 7}$ & 50 \\
\hline Solventes (\%) & $26 \%$ & $\mathbf{7 4 \%}$ & $100 \%$ \\
\hline
\end{tabular}

Pode-se notar que o modelo DEA atingiu o índice de acerto de $90 \%$ entre as empresas insolventes e de $74 \%$ entre as empresas solventes, o que resulta em $76,6 \%$ de acertos de classificação no total.

A Figura 2 mostra o comportamento dos escores de eficiência com relação à classificação das empresas entre solventes e insolventes, conforme a legenda à direita. A reta é o ponto de corte.

Figura 2: Comportamento dos Escores de Eficiência com Relação à Classificação das Empresas

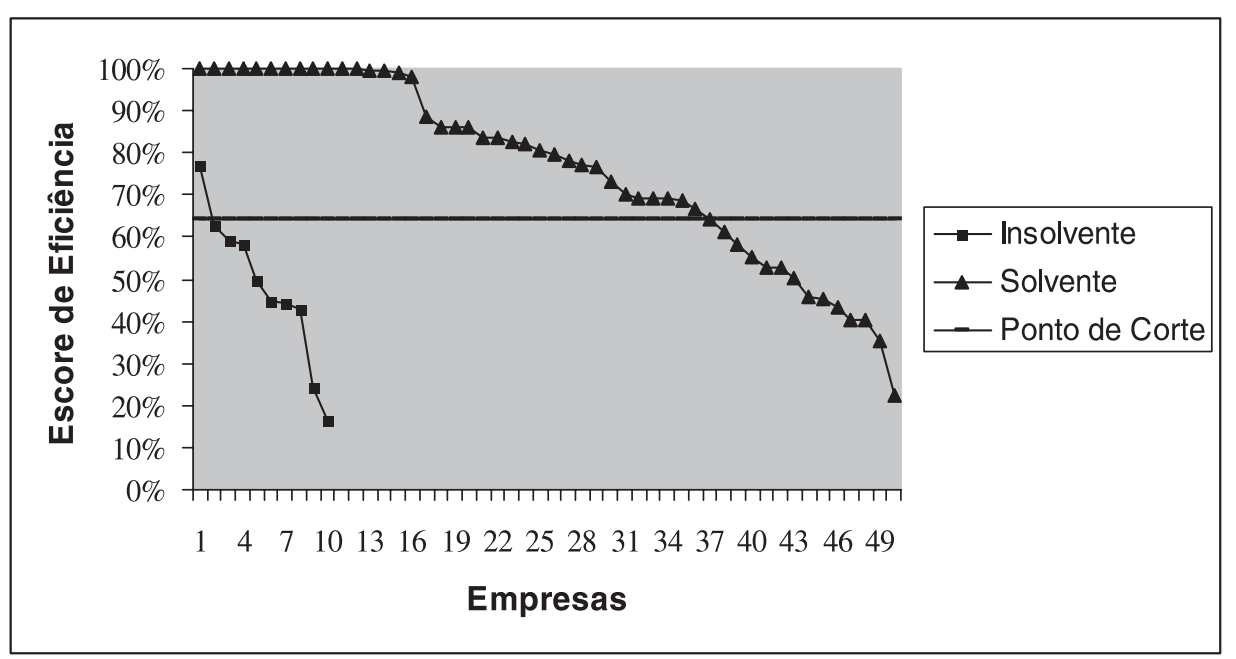

\section{Análises Estatísticas}

Análises adicionais contribuem para compreender os resultados obtidos pelo modelo DEA. A Tabela 5 mostra o resumo estatístico dos escores DEA, segregados entre empresas insolventes (Grupo 1) e solventes (Grupo 2). 
Tabela 5: Resumo Estatístico

\begin{tabular}{|r|r|r|r|r|r|r|r|r|}
\hline Grupo & N & Média & $\begin{array}{l}\text { Desvio- } \\
\text { Padrão }\end{array}$ & Mínimo & Q1 & Mediana & Q3 & Máximo \\
\hline 1 & 10 & 47,88 & 17,92 & 16,45 & 38,26 & 47,04 & 59,99 & 76,93 \\
\hline 2 & 50 & 76,40 & 21,66 & 22,22 & 60,40 & 80,16 & 99,69 & 100,00 \\
\hline
\end{tabular}

Legenda: Grupo 1 - Empresas Insolventes; Grupo 2 - Empresas Solventes

Percebe-se que a média dos escores para as empresas insolventes é menor do que para as empresas solventes. No entanto, o desvio-padrão para as empresas solventes é maior, mostrando maior dispersão dos escores em torno da média. A Figura 3 sintetiza as informações mostradas na Tabela 5 e realça as diferenças da distribuição dos escores entre os grupos de empresas.

\section{Figura 3: Boxplot dos Escores por Grupos de Empresas}

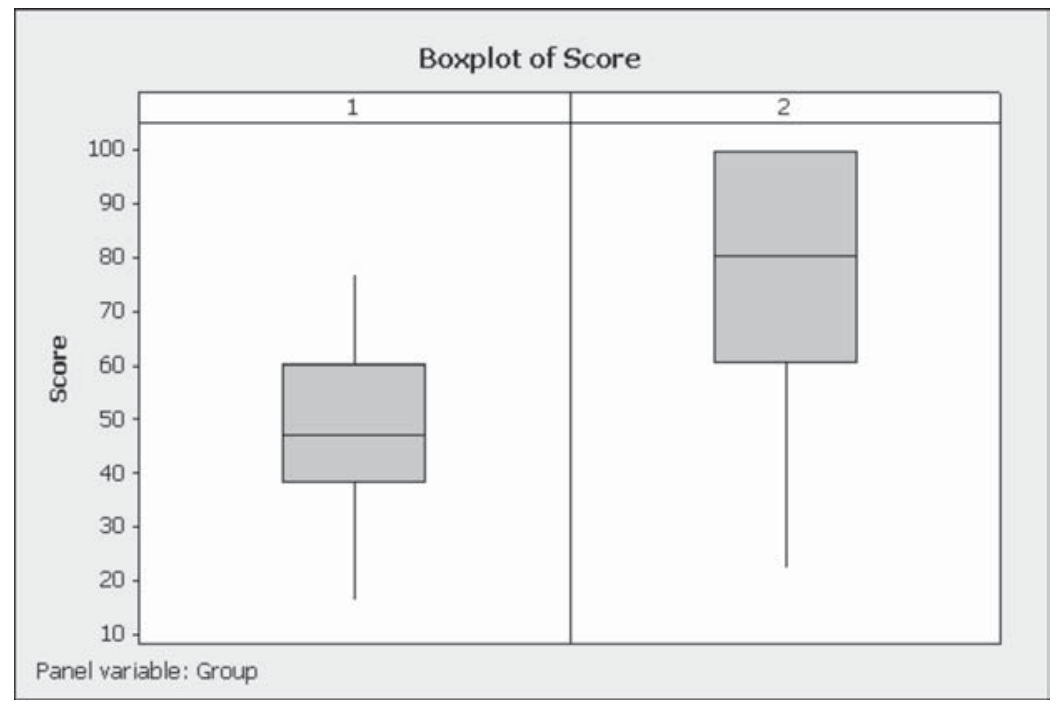

Legenda: Grupo 1 - Empresas Insolventes; Grupo 2 - Empresas Solventes

No gráfico Boxplot, o retângulo define o primeiro quartil (Q1), mediana e o terceiro quartil (Q3). As linhas estendem-se até:

. Limite Superior: Q1 - 1,5 (Q3 - Q1),

. Limite Inferior: Q3 + 1,5 (Q3 - Q1),

Se houvesse outliers (pontos extremos) seriam representados por asteriscos (*) distantes dos limites inferiores e superiores. 
Pode-se então concluir que os escores das empresas insolventes estão concentrados em valores inferiores aos das empresas solventes. Outro fato relevante é que não houve empresa insolvente que fosse considerada eficiente (escore $=100 \%)$. Pela Tabela 5 percebe-se que o escore máximo entre as empresas insolventes foi de 76,93.

O teste de hipótese de diferença entre as médias foi realizado e os resultados estão demonstrados na Tabela 6.

Tabela 6: Teste de Hipóteses: Escore versus Grupo

\begin{tabular}{|l|r|r|r|r|r|r|}
\hline Fonte & DF & \multicolumn{1}{l|}{ SS } & MS & F & P \\
\hline Grupo & 1 & 6775 & 6775 & 15,18 & 0,000 \\
\hline Error & 58 & 25881 & 446 & & \\
\hline Total & \multicolumn{2}{|l|}{59} & 32656 & & & \\
\hline$S=21,12$ & R-Sq $=20,75 \%$ & R-Sq(adj) $=19,38 \%$ & & & \\
\hline
\end{tabular}

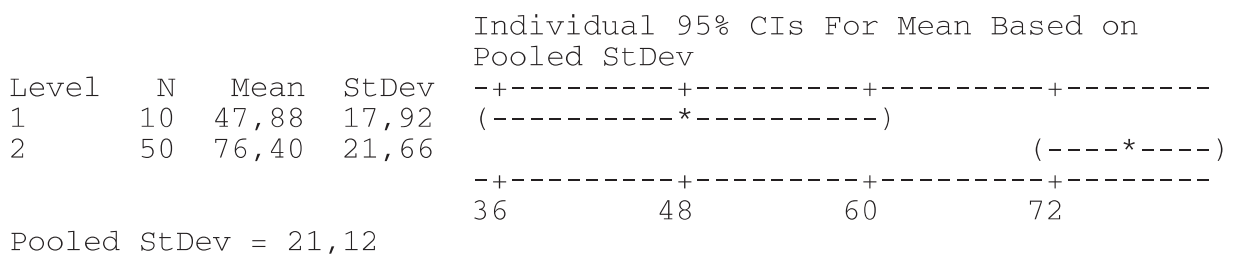

$\mathrm{O}$ valor $\mathrm{P}$ mostra que as diferenças dos escores médios entre os grupos são estatisticamente significativas.

As mesmas conclusões são obtidas aplicando o teste Kruskal-Wallis, indicado para amostras que não atendem à distribuição normal, cujos resultados são apresentados na Tabela 7.

Tabela 7: Teste Kruskal-Wallis: Escore versus Grupo

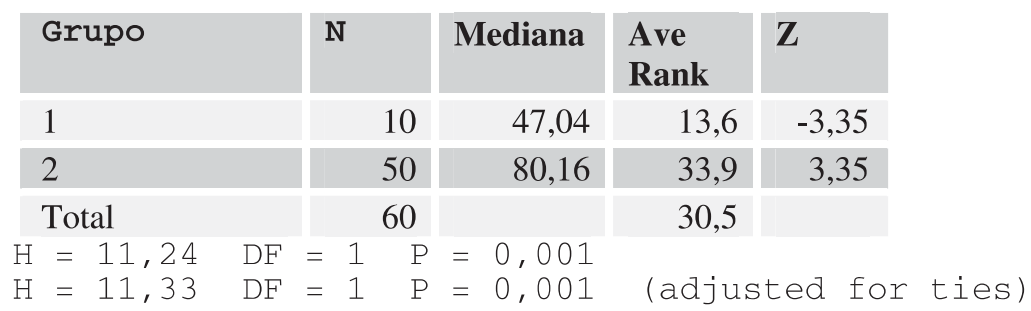




\section{Análises Adicionais}

Uma contribuição da análise DEA é a possibilidade de verificar os fatores que implicaram a determinação do escore de eficiência da empresa e quais as alterações permitiriam sua situação. Assim, uma possibilidade adicional apresentada pela DEA, em relação aos outros modelos de previsão de insolvência de empresas, é mostrar o que uma empresa insolvente poderia fazer para modificar sua situação para solvente.

É possível, por exemplo, comparar a atuação das 9 empresas posicionadas abaixo do ponto de corte, e classificadas corretamente como insolventes, com a das empresas classificadas como eficientes. De modo geral, com base neste confronto, percebe-se que as empresas insolventes deveriam diminuir seu endividamento e aumentar o crescimento de vendas e o retorno sobre o ativo.

Por outro lado, as 13 empresas solventes classificadas como insolventes têm um alto índice de endividamento, quando comparadas com as empresas eficientes. Por essa razão foram classificadas como ineficientes, implicando seu posicionamento abaixo do ponto de corte.

A Figura 4 apresenta um exemplo desta análise comparativa. Neste exemplo a empresa 6, classificada corretamente insolvente, apresentou o menor escore da amostra: $12,78 \%$. O modelo DEA comparou o desempenho da empresa 6 com a empresa 18 , considerada eficiente.

\section{Figura 4: Comparação de Empresa Insolvente com Empresa Eficiente}

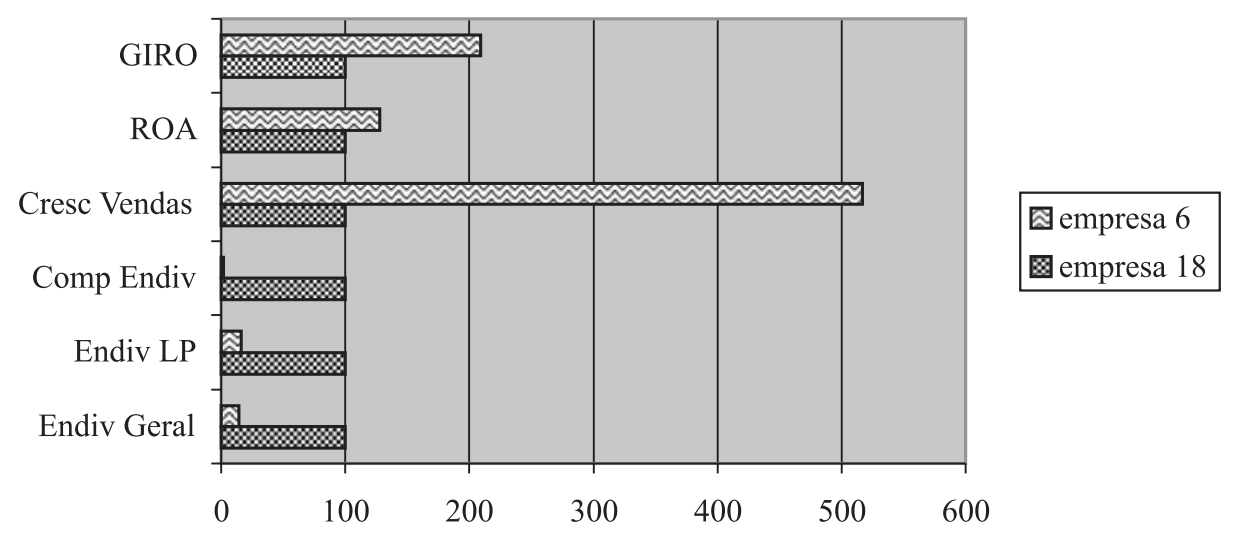

Pode-se notar que, em uma escala de 100 , a empresa 18 possui menor Endividamento (Geral e de Longo Prazo), maior Crescimento de Vendas, maior Retorno sobre o Ativo e maior Giro. 
Para a empresa 6 se tornar eficiente deveria reduzir seu Endividamento em $88 \%$, aumentar seu Crescimento de vendas em $299 \%$ e seu Giro em 64\%, como mostrado na Figura 5.

\section{Figura 5: Melhorias Sugeridas para a Empresa Insolvente}

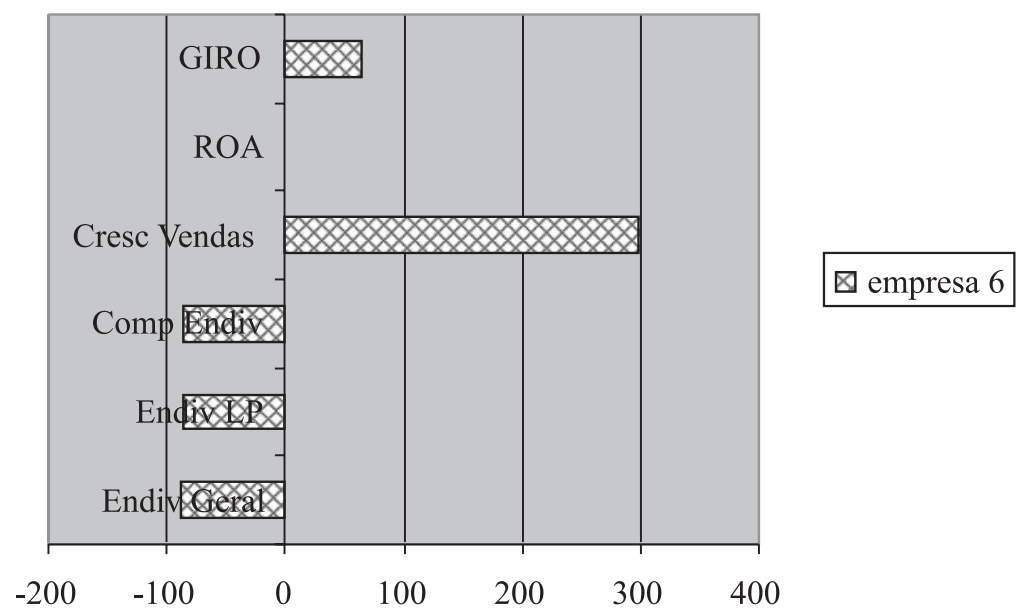

A possibilidade dessas análises adicionais proporcionadas pelo modelo DEA a tornam útil não somente para prever a insolvência, mas também para analisar possíveis alternativas de recuperação das empresas.

\section{Considerações Finais}

Os modelos de previsão de insolvência são de grande auxílio na avaliação de risco, sendo utilizados como ferramenta importante na análise de desempenho das empresas.

Este artigo teve como proposta a formulação de modelo baseado na Análise por Envoltória de Dados para previsão de insolvência de empresas. Para tanto foi construída uma base de dados composta por empresas que se tornaram insolventes e por uma amostra de controle, com empresas de mesmo porte, entre as de melhor desempenho do setor. Os indicadores contábeis foram analisados e incluídos em um modelo DEA com orientação ao insumo e retornos variáveis a escala (BCC-Insumo). Por assumirem valores negativos, as variáveis Crescimento de Vendas e Retorno sobre o Ativo sofreram transformação de escala, conforme previsto na literatura. 
Foram obtidas informações contábeis de três anos antes da insolvência das empresas e construído um modelo DEA. Foi determinado um ponto de corte, buscando-se a maior discriminação possível entre empresas solventes e insolventes. O resultado obtido com o modelo foi satisfatório, visto que classificou corretamente $90 \%$ das empresas insolventes presentes na amostra. O grau de acerto entre empresas solventes foi de $74 \%$. No total, as classificações corretas atingiram $76,6 \%$ dos casos.

Um aspecto relevante da técnica DEA é a possibilidade de comparar as empresas eficientes com as empresas ineficientes (benchmarking), indicando possibilidades de melhoria para seu desempenho. Para explorar essa possibilidade, uma empresa insolvente teve seu desempenho comparado com o de uma empresa solvente, considerada eficiente. Sugestões de melhoria foram apresentadas.

A pequena quantidade de indicadores contábeis foi uma das limitações da pesquisa. Outra limitação do estudo é que as conclusões se referem ao grupo de empresas selecionado, não podendo ser extrapoladas. O tamanho da amostra foi adequado às regras descritas na literatura sobre DEA. Porém outras pesquisas poderiam buscar uma expansão do número de empresas falidas/concordatárias.

Estudos futuros poderão dedicar-se a estudar procedimentos de seleção de variáveis e outros critérios para classificação das empresas entre solventes e insolventes. A aplicação conjunta de DEA com outras técnicas, como Análise Discriminante, Análise de Regressão Logística e Redes Neurais, também se constitui em uma possibilidade de estudo. O mesmo estudo pode ser replicado para empresas de outros setores e para outros períodos (panel data ou crosssectional). Outra possibilidade de estudo são modelos DEA com inclusão de variáveis macroeconômicas ou qualitativas.

\section{Artigo recebido em 24.05.2005. Aprovado em 16.09.2005.}

\section{REFERÊNCIAS BibliográficAs}

\section{Ali, A. I., \&}

Seiford, L. M. (1990).

Translation invariance in data envelopment analysis. Operations Research Letters, 9(6), 403-405.
Almeida, F. C. (1993).

L'Evaluation des risques de défaillance des entreprises à partir des réseaux de neurones insérés dans les systèmes d' aide à la décision. Thèse de doctorat, Ecole Supérieure des Affaires, Université Pierre Mendès France de Grenoble, France. 
Almeida, H. R., \&

Milioni, A. Z. (2000).

Análise de envoltória de dados na decisão de concessão de crédito. Anais do Simpósio Brasileiro de Pesquisa Operacional, Viçosa, MG, Brasil, 32.

Altman, E. L. (1968).

Financial ratios, discriminant analysis, and the prediction of corporate Insolventecy. Journal of Finance, 23(4), 589-609.

Altman, E.,

Haldeman, R., \&

Narayanan, P. (1977).

Zeta analysis. Journal of Banking and Finance, 1(1), 29-54.

Banker, R. D.,

Charnes, A., \&

Cooper, W. W. (1984).

Some models for estimating technical and scale inefficiencies in data envelopment analysis. Management Science, 30(9), 1078-1092.

Beaver, W. (1966).

Financial ratios as predictors of failure. Journal of Accounting Research, 4(Supplement), 71-111.

Bell, B. T.,

Ribar, G. R., \&

Verchio, J. R. (1990).

Neural nets vs. logistic regression: a comparison of each model's ability to predict commercial bank failures. Actes du congrès international de comptabilité (Tome 1), Nice, França.

Belloni, J. Â. (2000).

Uma metodologia de avaliação da eficiência produtiva de universi- dades federais brasileiras. Tese de doutorado, Departamento de Engenharia de Produção e Sistemas, Universidade Federal de Santa Catarina, SC, Brasil.

Ceretta, P. S. (1999).

Investigação empírica da eficiência do setor de alimentos. Gestão $e$ Produção, 6(3), 162-169.

Ceretta, P. S., \&

Niederauer, C. A. P. (2001).

Rentabilidade e eficiência do setor bancário brasileiro. Revista de Administração Contemporânea, 5(3), 7-26.

Charnes, A.,

Cooper, W. W.,

Lewin, A. Y., \&

Seiford, L. M. (Eds.). (1997).

Data envelopment analysis: theory, methodology, and application. Massachusetts, EUA: Kluwer.

Emel, A. B.,

Oral, M.,

Reisman, A., \&

Yolalan, R. (2003).

A credit score approach for the commercial banking sector. SocioEconomic Planning Sciences, 37(2), 103-123.

Exame (2003).

Critérios: indicadores. Anuário Melhores e Maiores. 38(12), 39-42.

Fernandez-Castro, A., \&

Smith, P. (1994).

Towards a general nonparametric model of corporate performance. Omega: International Journal of Management Science, 22(3), 237-249. 
Fitzpatrick, P. A. (1932).

A comparison of the ratios of the successful industrial enterprises with those of failed companies. Washigton: The Accountants Publishing Company.

Fitzsimmons, J. A., \&

Fitzsimmons, M. J. (2000).

Administração de serviços: operações, estratégias e tecnologia de informação (2a. ed.). (G. S. de Borba et al., Trad.) Porto Alegre: Bookman. (Obra original publicada em 1998).

Kanitz, S. C. (1974, dezembro). Como prever falências. Exame, pp.95103.

Laredo, A. (1997).

Modelos de previsão de insolvência baseados na análise de índices financeiros. Monografia de Graduação), Escola Superior de Propaganda e Marketing, São Paulo, SP, Brasil.

Matias, A. B. (1978).

Contribuição às técnicas de análise financeira: um modelo de concessão de crédito. Monografia de Graduação, Faculdade de Economia, Administração e Contabilidade, Universidade de São Paulo, SP, Brasil.

Niederauer, C. A. P. (1998).

Avaliação dos bolsistas de produtividade em pesquisa da engenharia da produção utilizando data envelopment analysis. Dissertação de Mestrado, Universidade Federal de Santa Catarina, Florianópolis, SC, Brasil.
Ohlson, J. A. (1980).

Financial ratios and the probabilistic prediction of Insolvency. Journal of Accounting Research, 18(1), 109-131.

Onusic, L. (2004).

A utilização conjunta das técnicas análise por envoltória de dados e regressão logística na previsão de insolvência de empresas: um estudo exploratório. Dissertação de mestrado, Universidade de São Paulo, SP, Brasil.

Paradi, J.,

Asmild, M., \&

Simak, P. (2004).

Using DEA and worst practice DEA in credit risk evaluation. Journal of Productivity Analysis, 21(2), 153-165.

Pastor, J. T. (1996).

Translation invariance in data envelopment analysis: a generalization. Annals of Operations Research, 66(2), 93-112.

Santos, A., \&

Casanova, S. P. C. (2005).

Proposta de um modelo estruturado de análise de demonstrações contábeis. RAE-Eletrônica [On-Line], 4(1), 1-27. Recuperado de http:// w w w. r a e. c o m . b r / eletronicaindex.cfm?FuseAction= Artigo \& ID $=1696 \& \mathrm{Sec}$ a o $=$ ARTIGOS\&Volume $=4 \&$ numero $=$ $1 \&$ Ano $=2005$

Silva, J. P. da (1997).

Gestão e análise de risco de crédito.

São Paulo: Atlas. 
Simak, P.C. (1997).

Dea based analysis of corporate failure. Thesis of Master, Graduate Department of Mechanical and Industrial Engineering, University of Toronto, Toronto, Canada.

Simak, P. C. (2000).

Inverse and negative DEA and Their application to credit risk evaluation. Thesis of Doctor, Graduate Department of Mechanical and Industrial Engineering, University of Toronto, Toronto, Canada.

Smith, P. (1990).

Data envelopment analysis applied to financial statements. Omega International Journal of Management Science, 18(2), 131-138.
Tam, K. Y., \&

Kiang, M. Y. (1992).

Managerial applications of neural networks: the case of bank failure predictions. Management Science, 38(7), 913-925.

Zhu, J. (2000).

Multi-factor performance measure model with an application to Fortune 500 companies. European Journal of Operational Research, 123(1), 105124.

Zhu, J. (2003).

Quantitative models for performance evaluation and benchmarking: data envelopment analysis with spreadsheets and DEA Excel Solver. Boston: Kluwer Academic. 
